\section{De la pâleur au bronzage. Les idéaux de la beauté féminine en France}

\author{
Peter Frost
}

a peau glabre recouvre la quasi-totalité du corps humain ; elle domine ainsi la perception de soi et des autres. C'est pourquoi nous apprécions tant ses propriétés tangibles et visuelles, comme sa douceur, sa nature lisse et sa couleur. Si cette dernière indique aujourd'hui surtout l'origine ethnique, elle permettait autrefois, au sein d'une même ethnie, de différencier les individus par sexe et par âge.

\section{Repères biologiques et culturels}

Tous les êtres humains naissent pâles ; ce fait est bien connu chez les peuples à la peau foncée qui y voient une marque du nouveau-né [Maertens, 1978, p. 41; Walentowitz, 2008 ; Zahan, 1974]. Au stade adulte, la pâleur distingue aussi la femme, dont l'épiderme est moins pourvu en mélanine et en hémoglobine, ce qui lui donne un teint plutôt pâle par rapport au rouge-brun de l'homme [Edwards et Duntley, 1939]. C'est au cours de l'adolescence que le teint féminin se différencie du teint masculin, en s'éclaircissant sous l'influence des hormones sexuelles [Edwards et Duntley, 1949 ; Edwards et al., 1941 ; Kalla et Tiwari, 1970 ; Mesa, 1983]. Cet éclaircissement est moindre aux deux extrêmes de la pigmentation humaine, soit la peau très pâle et la peau très foncée. C'est donc chez les peuples de couleur moyenne que le teint féminin s'éclaircit le plus par rapport au teint masculin [Frost, 2007 ; Madrigal et Kelly, 2006].
Pourquoi ces différence: croient que le corps de la fe: aspects clés - sa pâleur, son douce et glabre. Selon eux l'homme et à lui soutirer les. [Blaffer-Hrdy, 2000, p. 445 1986]. Mais peu importe la relative, bien plus que la fo: individu est une femme et inconsciente [Dupuis-Roy e 2007 ; Tarr et al., 2001].

Aujourd'hui, ces petites sont moins visibles, notamm par notre fréquentation des ment effacées par la mode fé: tion plutôt récente, et il fa: suscitait autrefois un plus gra

Cet intérêt s'était surtout allant de la Méditerranée à la table culte de la blancheur $f$ moyenne, le teint féminin s plus, cette zone avait été le b. premiers efforts pour mettre de beauté. En appliquant ce divers moyens (chapeau à gro prochait davantage de l'idé précisait avec l'essor des art objet de quête, voire d'encha

Le cas de la Franc
Cette zone eurasiati
peuple connu dans l'Antiquit:
de Pétrone nous présente des
teignent à l'encre noire pour
lise: « Devient-on gaulois en s
tard, on continuait de soulig
royaume des Francs dans l'Es
ou des concubines: " Ces Fra
qu'elles étaient blondes et de 
Pourquoi ces différences d'âge et de sexe ? Certains évolutionnistes croient que le corps de la femme imite celui du nourrisson sous plusieurs aspects clés - sa pâleur, son "visage de bébé ", sa voix aiguë et sa peau douce et glabre. Selon eux, cette allure enfantine servirait à apaiser l'homme et à lui soutirer les soins qu'exige la lente maturation de l'enfant [Blaffer-Hrdy, 2000, p. 445 ; Guthrie, 1970; Van den Berghe et Frost, 1986]. Mais peu importe la cause ultime, nous savons que cette pâleur relative, bien plus que la forme du visage, nous aide à déterminer qu'un individu est une femme et non un homme, et ce souvent de manière inconsciente [Dupuis-Roy et al., 2009 ; Russell, 2010 ; Russell et Sinha, 2007 ; Tarr et al., 2001].

Aujourd'hui, ces petites différences de teint entre hommes et femmes sont moins visibles, notamment parce qu'elles sont de plus en plus noyées par notre fréquentation des peuples du monde entier; elles sont également effacées par la mode féminine du bronzage. Mais il s'agit d'une situation plutôt récente, et il faut savoir que la pâleur relative de la femme suscitait autrefois un plus grand intérêt.

Cet intérêt s'était surtout développé dans une vaste zone eurasiatique allant de la Méditerranée à la mer de Chine, y donnant naissance à un véritable culte de la blancheur féminine. D'abord, la peau y étant de couleur moyenne, le teint féminin s'éclaircissait davantage après la puberté. De plus, cette zone avait été le berceau des premières civilisations et donc des premiers efforts pour mettre en valeur la féminité, parmi lesquels les soins de beauté. En appliquant certains fards et en se protégeant du soleil par divers moyens (chapeau à grands bords, gants, ombrelle), la femme se rapprochait davantage de l'idéal du teint blanc. Parallèlement, cet idéal se précisait avec l'essor des arts picturaux et littéraires, devenant ainsi un objet de quête, voire d'enchantement.

\section{Le cas de la France}

Cette zone eurasiatique longeait par le sud le pays des Gaulois, un peuple connu dans l'Antiquité pour la pâleur de sa peau. Ainsi, le Satiricon de Pétrone nous présente des personnages qui, en cherchant à se cacher, se teignent à l'encre noire pour paraître éthiopiens ; cependant, on les ridiculise : «Devient-on gaulois en se blanchissant à la craie ? » Un millénaire plus tard, on continuait de souligner la blancheur des captives ramenées du royaume des Francs dans l'Espagne musulmane pour en faire des épouses ou des concubines : "Ces Franques d'autant plus recherchées à Cordoue qu'elles étaient blondes et de teint clair » [Lévi-Provençal, 1953, p. 179]. 
Mais c'étaient là des regards extérieurs. Au Moyen Âge, seule une minorité de Français se décrivait comme «blanche »; les autres se disaient «bruns » ou même «noirs » - c'était ainsi qu'on représentait celui à la peau basanée, qu'il fût berger, brigand, laideron ou Sarrasin [Colby, 1965, p. 82, 85-86]. Ces couleurs se répartissaient dans la population française sans doute un peu comme les noms de famille correspondants aujourd'hui : $36 \%$ (Leblanc), $39 \%$ (Lebrun), $25 \%$ (Lenoir) [Tous les noms de famille, 2012]. On pourrait objecter que ces patronymes indiquaient la couleur de la chevelure ; mais les cheveux blancs n'étaient pas aussi fréquents que les cheveux bruns, surtout lorsque la durée de vie moyenne ne dépassait pas quarante ans...

Sur le fond de ces différences entre familles se dressait celle distinguant la femme de l'homme. Son importance se voit dans les prénoms féminins évoquant la blancheur, comme Aurore, Blanche, Blanchefleur, Claire, Hermine, Marguerite ou Margot, ainsi que dans la peinture, où les images de femmes étaient toujours plus blanches que les images d'hommes [Phan et Flandrin, 1984]. Cette convention artistique est attestée par toutes les civilisations connaissant l'art pictural : grécoromaine, égyptienne, chinoise, japonaise, aztèque, etc. [Capart, 1905, p. 26-27 ; Soustelle, 1955, p. 159 ; Wagatsuma, 1967].

Passons aux soins de beauté, dont la plupart servaient à blanchir la peau. Il existait surtout des poudres d'origine végétale ou minérale (riz, amidon, fleur de froment, plâtre, céruse), ainsi que des préparations visant à réduire la pigmentation elle-même ou à éliminer les taches, les rougeurs, les rousseurs et les grains de beauté (jus de citron, mercure, plomb). On disait alors vouloir « albifier " la peau féminine, l'éclaircir, la clarifier, la pâlir... [Lanoë, 2007].

Dans la littérature, enfin, la femme est décrite par toute une symbolique de la blancheur, comme l'hermine, la neige, le lys ou la fleur d'aubépine [Colby, 1965, p. 4, 37-67]. C'est ainsi que le roman Aucassin et Nicolette dépeint son héroïne : «Les fleurs des marguerites, que ses pieds brisaient au passage et qui retombaient sur eux étaient toutes noires auprès de ses pieds et de ses jambes, tant était blanche la fillette. » Lorsque Nicolette veut se faire passer pour un jongleur, donc pour un homme, elle s'habille en tunique et culotte, puis assombrit son visage [Aucassin et Nicolette, 1964].

Si cette évocation de la blancheur n'est souvent qu'une mise en scène, il arrive qu'on l'insère dans la trame même du récit. Dans Perceval le Gallois, de Chrétien de Troyes, le héros voit un faucon abattre une oie, dont trois gouttes de sang tombent sur la neige : "Quand il vit la neige tassée à l'endroit et le sang tout autour, il s'appuya sur sa lance pour regarder cette apparence étran rappellent les vives couleurs $d$ tiers qu'il oublie où il est. Com tait sur le blanc, ainsi les tr blancheur de la neige " [Chrét

Ce motif du sang sur la ne écrites d'une zone qui déborde et en Asie centrale [Cosquin, 1 et parfois un souhait. Ainsi, reine qui, un jour d'hiver, se $p$ scène l'émeut : « Oh! si je pour aussi vermeil que le sang et a fenêtre! » Peu après, elle dor Grimm, 1967, p. 299]. Dans blanche peut devenir une fem. sculpture en marbre, tantôt tasme nous donne la femme-c klore d'une vaste zone eurasia

Si la référence à la peau c femme, il existait néanmoins gnaient des ballades paysann celle du discours dominant. femme à l'idée d'un amour objet de passion subite. Ainsi, sitôt soupçonnée d'avoir e [Massny, 1937 ; Vasvari, 199 ? niques animaient de nomb: «bonne " et «pure» est « ardente " et "passionnée " blonde finit mariée, la brune,

\section{La mode du bronz}

Au début du $\mathrm{XX}^{e}$ sil libéré des contraintes de la tr de l'éradication par les rayon qui a incité la communauté n sous la lampe UV. La nouvell a vu un moyen de préventio d'abord pratiqués discrèteme 
regarder cette apparence étrange : le sang et la neige ainsi rapprochés lui rappellent les vives couleurs de Blanchefleur son amie. Il y pense si volontiers qu'il oublie où il est. Comme en la face de son amie le vermeil ressortait sur le blanc, ainsi les trois gouttes de sang se détachent sur la blancheur de la neige » [Chrétien de Troyes, 1947, p. 98].

Ce motif du sang sur la neige se retrouve dans les traditions orales et écrites d'une zone qui déborde l'Europe pour s'étendre en Afrique du Nord et en Asie centrale [Cosquin, 1922]. Il sert souvent à évoquer un souvenir et parfois un souhait. Ainsi, par exemple, un conte nous présente une reine qui, un jour d'hiver, se pique le doigt devant une fenêtre d'ébène. La scène l'émeut : « Oh ! si je pouvais avoir un enfant aussi blanc que la neige, aussi vermeil que le sang et aussi noir de cheveux que l'ébène de cette fenêtre ! » Peu après, elle donne naissance à Blanche-Neige [Grimm et Grimm, 1967, p. 299]. Dans le même ordre d'idées, un objet de couleur blanche peut devenir une femme. L'objet est tantôt inanimé, comme une sculpture en marbre, tantôt animé, comme un oiseau. Ce dernier fantasme nous donne la femme-cygne, qui se retrouve également dans le folklore d'une vaste zone eurasiatique [Thompson, 1975, p. 34, 61].

Si la référence à la peau claire dominait le discours érotique sur la femme, il existait néanmoins un érotisme de la peau brune, dont témoignaient des ballades paysannes européennes et dont la logique inversait celle du discours dominant. À l'encontre du teint blanc, qui associe la femme à l'idée d'un amour durable, le teint foncé la transforme ici en objet de passion subite. Ainsi, si une jeune fille se met à brunir, elle est aussitôt soupçonnée d'avoir eu des rapports sexuels avant le mariage [Massny, 1937 ; Vasvari, 1999]. À la Belle Époque, ces érotismes antagoniques animaient de nombreux romans, où la blonde "innocente", "bonne " et "pure " est dressée contre la brune «impétueuse", " ardente » et "passionnée »-d'une féminité dévorante en somme. Si la blonde finit mariée, la brune, elle, est délaissée [Atzenhoffer, 2011].

\section{La mode du bronzage}

Au début du $\mathrm{xx}^{\mathrm{e}}$ siècle, cet érotisme alternatif s'est peu à peu libéré des contraintes de la tradition. Tout a commencé par la découverte de l'éradication par les rayons ultraviolets du bacille de la tuberculose, ce qui a incité la communauté médicale à mettre les tuberculeux au soleil ou sous la lampe UV. La nouvelle thérapie a aussi rallié le grand public, qui y a vu un moyen de prévention et même une panacée. Les bains de soleil, d'abord pratiqués discrètement, se sont faits de plus en plus au grand jour, 
amenant certains adeptes au naturisme. Enfin, une percée s'est effectuée au cours des années 1920, à tel point que le magazine Vogue a proclamé à la fin de la décennie que « The 1929 girl must be tanned» (« La femme de 1929 doit être bronzée ») [Andrieu, 2008 ; Bard, 1998, p. 41 ; Frost, 2010, p. 91-102; Segrave, 2005]. La peau bronzée, qui n'était dans un premier temps que l'effet secondaire de l'exposition au soleil, est devenue un but, voire une mode. C'est la styliste Gabrielle (Coco) Chanel qui l'aurait lancée, en 1925 dit-on, en affichant un teint bronzé après un séjour sur le yacht du duc de Westminster. En réalité, cette mode l'a précédée, comme en témoigne par exemple le roman Le Blé en herbe, publié en 1924 : «Vinca l'attendait sur le pré de mer et cuisait paisiblement au soleil ses hautes jambes, ses bras déliés d'un brun roux de pain campagnard » [Colette, 1969, p. 109-110].

Cette nouvelle manie suscitait l'étonnement. En 1929, une journaliste du New York Times faisait ainsi remarquer : si c'est pour des raisons de santé qu'on bronze avec une telle ardeur, pourquoi ne pas mettre autant d'énergie à manger des épinards, à dormir ou à mettre des souliers orthopédiques? [Segrave, 2005, p. 35]. En effet, les présumées vertus thérapeutiques expliquent mal la soudaine popularité du bronzage, et moins encore sa métamorphose en mode féminine. Notons qu'il existait au même moment d'autres tendances de la mode, également étonnantes, qu'on regroupe sous le vocable de la « garçonne ", titre d'un roman à scandale paru en 1922. La garçonne présentait une allure androgyne : cheveux coupés court, épaules larges, poitrine plate, hanches étroites et jambes longues [Bard, 1998]. Ces nouveaux éléments, tout comme le bronzage, ont permis d'explorer de nouveaux érotismes en masculinisant la figure féminine.

De manière générale, la mode du bronzage témoigne d'une certaine vision de la femme, tout comme le culte de la blancheur qui la précédait. Elle incarne un nouvel érotisme qui fascine autant l'observateur que l'observée, en créant un nouveau champ d'attraction. Si, depuis une trentaine d'années, cette mode n'existe plus dans certains pays, comme le Japon, un retour à la norme traditionnelle tarde à arriver en Europe [Ashikari, 2005]. C'est peut-être parce que la pâleur féminine évoque trop l'image d'un être vulnérable qu'il faut protéger, que ce soit un nourrisson ou une jeune mère. Cette image, considérée comme dépassée, devient donc aussi un obstacle à la jouissance...
ASHIRARI M. (2005), * Culti cosmetics boom and the $]$ : vol. 10, p. 73-91.

Aucassin et Nicolette (1964 Bocard, Paris.

ANDRIEU B. (2008), Bromzas? Éditions, Paris.

ATZENHOFFER R. (2011), < I aussi. Effet de psycholog héroïnes dans l'cuvre ro: Colloque national (CNRI) http://cnriut09.univ-lille BARD C. (1998), Les Gargor Flammarion, Paris.

BLAFFER-HRDY S. (2000), M Shape the Human Species, CAPART J. (1905), Primitive Chrétien De Troyes (1947) Paris.

Colby A. M. (1965), The F Genève.

COLETTE (1969), Le Bléen: Cosquin E. (1922), Les Co Paris.

Dupuis-Roy N., Fortin L, discrimination cues ina p. 1-8.

EDWARDS E. A. et DUNTLE: women in reference to th Journal of Obstetrics and $C$

- (1939), « The pigments Joumal of Anatomy, vol 6

EDWARDS E. A., Hamiltos vascular and pigmentar Endocrinology, vol. 28, p

Frost P. (2010), Femmes colorisme, Presses de l'ur - (2007), « Comment on the sexual selection hyp Anthropology, vol. 133, p 


\section{Bibliographie}

ASHIKARI M. (2005), “ Cultivating Japanese whiteness. The "whitening" cosmetics boom and the Japanese identity », Journal of Material Culture, vol. 10, p. 73-91.

Aucassin et Nicolette (1964), in Poèmes et récits de la vieille France, E. De Bocard, Paris.

ANDRIEu B. (2008), Bronzage. Une petite histoire du Soleil et de la peau, CNRS Éditions, Paris.

AtzenHoffer R. (2011), « Les hommes préfèrent les blondes. Les lectrices aussi. Effet de psychologie, horizons idéologiques et valeurs morales des héroïnes dans l'œuvre romanesque de Hedwig Courths-Mahler ", Colloque national (CNRIUT), Villeneuve-d'Ascq, 8-10 juin 2009, http://cnriut09.univ-lille1.fr.

BARD C. (1998), Les Garçonnes. Modes et fantasmes des Années folles, Flammarion, Paris.

BLAFFER-Hrdy S. (2000), Mother Nature. Maternal Instincts and How They Shape the Human Species, Ballantine Books, New York.

Capart J. (1905), Primitive Art in Egypt, H. Grevel, Londres.

Chrétien de Troyes (1947), Perceval le Gallois ou le conte du Graal, Stock, Paris.

ColBY A. M. (1965), The Portrait in Twelfth-Century French Literature, Droz, Genève.

COLETTE (1969), Le Blé en herbe, Garnier-Flammarion, Paris.

Cosquin E. (1922), Les Contes indiens et l'Occident, Honoré Champion, Paris.

Dupuis-Roy N., Fortin I., Fiset D. et al. (2009), « Uncovering gender discrimination cues in a realistic setting ", Journal of Vision, vol. $9, \mathrm{n}^{\circ} 2$, p. 1-8.

Edwards E. A. et DunTley S. Q. (1949), « Cutaneous vascular changes in women in reference to the menstrual cycle and ovariectomy ", American Journal of Obstetrics and Gynecology, vol. 57, p. 501-509.

- (1939), «The pigments and color of living human skin », American Journal of Anatomy, vol. 65, p. 1-33.

Edwards E. A., Hamilton J. B. et DunTley S. Q. etal. (1941), « Cutaneous vascular and pigmentary changes in castrate and eunuchoid men ", Endocrinology, vol. 28, p. 119-128.

Frost P. (2010), Femmes claires, hommes foncés. Les racines oubliées $d u$ colorisme, Presses de l'université Laval, Québec.

- (2007), "Comment on human skin-color sexual dimorphism. A test of the sexual selection hypothesis », American Journal of Physical Anthropology, vol. 133, p. 779-781. 
-(1988), « Human skin color. A possible relationship between its sexual dimorphism and its social perception $"$, Perspectives in Biology and Medicine, vol. 32, p. 38-58.

Grimm J. et GRIMM W. (1967), Les Contes, Flammarion, Paris.

GUTHRIE R. D. (1970), « Evolution of human threat display organs ", Evolutionary Biology, vol. 4, p. 257-302.

KALLA A. K. et TrWARI S. C. (1970), « Sex differences in skin colour in man », Acta Geneticae Medicae et Gemellologiae, vol. 19, p. 472-476.

LANOË C. (2007), « L'invention de la peau. Les techniques de blanchiment đu visage à l'époque moderne, XVI ${ }^{\mathrm{e}}$-XviIr siècle », Communications, $\mathrm{n}^{\circ} 81$, p. 107-120.

LÉvI-ProvenÇAL É. (1953), Histoire de l'Espagne musulmane, t. III, Éditions G. P. Maisonneuve, Paris.

MADRIGAL L. et KelLy W. (2006), « Human skin-color sexual dimorphism: A test of the sexual selection hypothesis ", American Journal of Physical Anthropology, vol. 132, p. 470-482.

MAERTENS J.-T. (1978), Ritologiques, t. I, Le Dessein sur la peau. Essai d'anthropologie des inscriptions tégumentaires, Aubier Montaigne, Paris. MASSNY D. (1937), «Die Formel "das braune Mägdelein" im alten deutschen Volkslied », Niederdeutsche Zeitschrift für Volkskunde, vol. 15, p. 26-65.

MESA M. S. (1983), « Analyse de la variabilité de la pigmentation de la peau durant la croissance », Bulletin et mémoires de la Société d'anthropologie de Paris, vol. 10, $\mathrm{n}^{\circ} 10-11$, p. 49-60.

Phan M. C. et Flandrin J.-L. (1984), « Les métamorphoses de la beauté féminine ", L'Histoire, vol. 68, p. 48-57.

RusSell R. (2010), "Why cosmetics work ", in AdAMs R. B., AMBADY N., NARAYAMA K. et al. (dir.), The Science of Social Vision, Oxford University Press, New York.

- (2003), «Sex, beauty, and the relative luminance of facial features », Perception, vol. 32, p. 1093-1107.

RUSSELL R. et SinHA P. (2007), « Real-world face recognition. The importance of surface reflectance properties », Perception, vol. 36, p. 1368-1374.

Segrave K. (2005), Suntanning in 20 $0^{\text {th }}$ Century America, McFarland \& Co, Jefferson.

Soustelle J. (1955), La Vie quotidienne des Aztèques, Hachette, Paris.

Tarr M. J., Kersten D., Cheng Y. et al. (2001), «It's Pat ! Sexing faces using only red and green », Journal of Vision, vol. 1, $\mathrm{n}^{\circ} 3$, p. 337-337a.

Thompson S. (1975), Motif-Index of Folk-Literature, vol. 2, Indiana University Press, Bloomington et Londres.
«Tous les noms de www.nom-famille VAN DEN BERGHEP.I dimorphism, and s co-evolution ? $s, \mathrm{E}$ VASVARI L. O. (1999 and the erotic wed Culture, vol. 1, déce WAGATSUMA H. (190 Daedalus, vol. 96, F WALENTOWITZ S. ( 20 et de la pigmentati. BLANCHARD P. et al. ZAHAN D. (1972), * Africa ", in Pontmay p. 365-395. 
«Tous les noms de famille» (2012), site auFeminin.com, www.nom-famille.com.

VANDEN BERGHe P. L. et Frost P. (1986), « Skin color preference, sexual dimorphism, and sexual selection. A case of gene-culture co-evolution? ", Ethnic and Racial Studies, vol. 9, p. 87-113.

VASVARI L. O. (1999), «A comparative approach to European folk poetry and the erotic wedding motif ", CLCWeb. Comparative Literature and Culture, vol. 1, décembre.

WAGATSUMA H. (1967), "The social perception of skin color in Japan », Daedalus, vol. 96, p. 407-443.

WALENTOWITZ S. (2008), « Des êtres à peaufiner. Variations de la coloration et de la pigmentation du nouveau-né », in ALberT J.-P., ANDrieu B., BlanChard P. et al. (dir.), Coloris Corpus, CNRS Éditions, Paris, p. 113-120. ZAHAN D. (1972), «White, red and black. Colour symbolism in black Africa ", in Portmann A. et RITSEMA R. (dir.), The Realms of Colour, vol. 41, p. 365-395. 